\title{
Tunable Dirac cones in two-dimensional acoustic metamaterials with matryoshka structure
}

Meng Chen, Wenshuai Xu, Yu Liu, Mangong Zhang, Dongliang Pei, Heng Jiang, and Yuren Wang

Citation: The Journal of the Acoustical Society of America 146, 767 (2019); doi: 10.1121/1.5115007

View online: https://doi.org/10.1121/1.5115007

View Table of Contents: https://asa.scitation.org/toc/jas/146/1

Published by the Acoustical Society of America

\section{ARTICLES YOU MAY BE INTERESTED IN}

A comparison study between acoustic topological states based on valley Hall and quantum spin Hall effects The Journal of the Acoustical Society of America 146, 721 (2019); https://doi.org/10.1121/1.5115017

Reconfigurable topological insulator for elastic waves

The Journal of the Acoustical Society of America 146, 773 (2019); https://doi.org/10.1121/1.5114920

Acoustic valley edge states in a graphene-like system with sub-wavelength resonator

The Journal of the Acoustical Society of America 146, 736 (2019); https://doi.org/10.1121/1.5115016

Spectral analysis of amplitudes and phases of elastic waves: Application to topological elasticity The Journal of the Acoustical Society of America 146, 748 (2019); https://doi.org/10.1121/1.5114911

Pseudospins and topological edge states for fundamental antisymmetric Lamb modes in snowflakelike phononic crystal slabs

The Journal of the Acoustical Society of America 146, 729 (2019); https://doi.org/10.1121/1.5114903

Non-reciprocal wave phenomena in energy self-reliant gyric metamaterials

The Journal of the Acoustical Society of America 146, 789 (2019); https://doi.org/10.1121/1.5114916

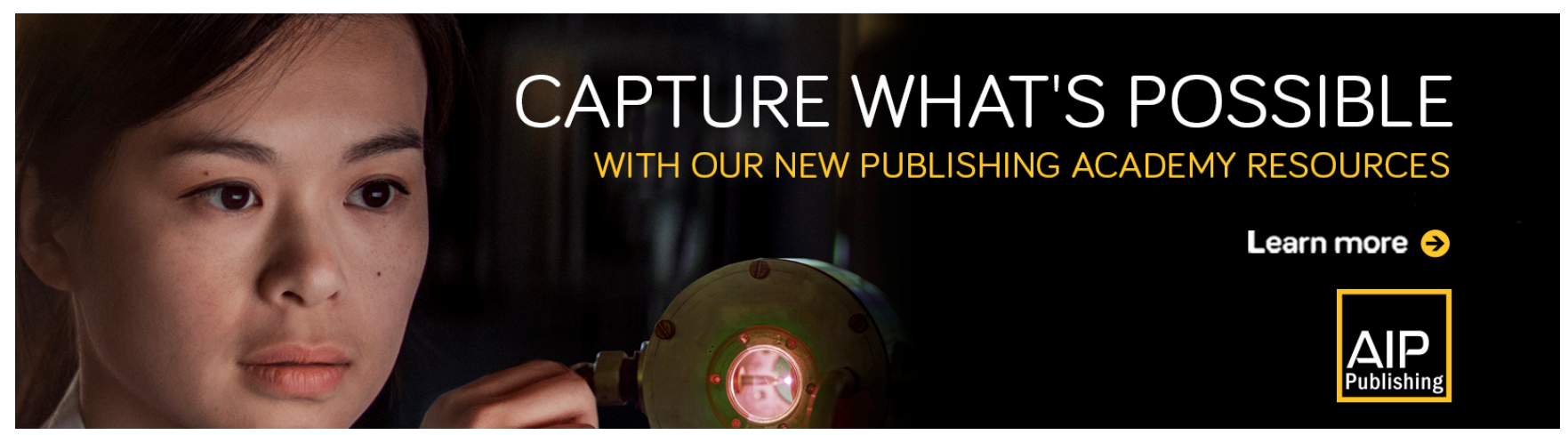




\title{
Tunable Dirac cones in two-dimensional acoustic metamaterials with matryoshka structure
}

\author{
Meng Chen, ${ }^{1, a)}$ Wenshuai $\mathrm{Xu},{ }^{1, a)}$ Yu Liu, ${ }^{1, a)}$ Mangong Zhang, ${ }^{2}$ Dongliang Pei, ${ }^{1, a)}$ \\ Heng Jiang, ${ }^{1, a)}$ and Yuren Wang ${ }^{1, b)}$ \\ ${ }^{1}$ Key Laboratory of Microgravity, Institute of Mechanics, Chinese Academy of Sciences, Beijing 100190, \\ People's Republic of China \\ ${ }^{2}$ Wuhan Second Ship Design and Research Institute, Wuhan, Hubei 430064, People's Republic of China
}

(Received 8 January 2019; revised 20 April 2019; accepted 9 May 2019; published online 31 July 2019)

\begin{abstract}
Dirac cones of an acoustic system are the foundation of most topological phase transitions and topological states and have recently become a research hotspot. Although the Dirac cones, Dirac-like cones, double Dirac cones, and semi-Dirac points are all skillfully designed, it is still indispensable to realize a tunable Dirac cone in a novel acoustic structure. This paper proposes two-dimensional acoustic metamaterials with matryoshka structure to achieve tunable Dirac cones and topological spin states. Dirac points can be obtained on the dispersion curves owing to the high symmetry. The concentric circular scattering units of the matryoshka structure are arranged in honeycomb lattices. By a rotating-scatterer mechanism to break the symmetry, the Dirac cone at $K\left(K^{\prime}\right)$ is split and the topological spin states appear at the band valley. The existence of a topological transition with opposite Chern numbers as the rotating angle varies is also verified, and helical edge states are obtained along the interfaces separating the topologically opposite spin states insulators. Moreover, the frequency of the Dirac cone is tuned by rotating the inner structure in a double-layer matryoshka structure. (C) 2019 Acoustical Society of America. https://doi.org/10.1121/1.5115007
\end{abstract}

$[\mathrm{GH}]$

Pages: $767-772$

\section{INTRODUCTION}

In recent years, the topological states and the physical phenomena of acoustic systems have gradually become research hot spots (Ma and Sheng, 2016; Wu et al., 2018). Because the acoustic topology protection of boundary state transmission can effectively suppress backscattering and has a strong robustness to defects (He et al., 2016; Lu et al., 2016; Wang et al., 2015), it has great application prospects in acoustic communication, noise control, and so on. Analogous to electronic systems, the topological phase transition and robust boundary states in band gap structures can be excited by breaking the time-reversal symmetry (Fleury et al., 2014; Khanikaev et al., 2015; Yang et al., 2015), using temporal (or spatial) modulation (Fleury et al., 2016) and coupling resonance (He et al., 2016; Peng et al., 2016; Wei et al., 2017) or reducing the space symmetry (Dai et al., 2017; Lu et al., 2014; Lu et al., 2016; Wen et al., 2018; Xia et al., 2017; Xia et al., 2018; Xia et al., 2019; Zhang et al., 2017) in artificial periodic structures, such as sonic crystals. The key to realizing an acoustic topological phase transition is to degrade the dispersion relation of Dirac cones by introducing additional degrees of freedom, so as to obtain the spin states and then construct the acoustic topological insulator (Li et al., 2014). Therefore, the formation of Dirac cones in a band structure is the foundation of most topological phase transitions and topological states.

\footnotetext{
a) Also at: University of Chinese Academy of Sciences, Beijing, 100049, People's Republic of China.

b) Also at: University of Chinese Academy of Sciences, Beijing, 100049, People's Republic of China. Electronic mail: yurenwang@imech.ac.cn
}

Dirac cones refer to the central point of the degeneracy states with linear dispersion in momentum space at the high symmetry point of a Brillouin zone (BZ) boundary, which originates from the study of the band structure of monolayer grapheme (Häusler and Egger, 2009; Novoselov et al., 2005; Zhang et al., 2005). The Dirac cone can be generated by either deterministic degeneracy, arising from the consequence of the high crystal symmetry, or accidental degeneracy, owing to fine-tuning parameters (including external fields or structure parameters) (Herring, 1937). Current studies show that Dirac cones in acoustic systems can be divided into a Dirac cone (with twofold Dirac degeneracy) (Lu et al., 2014; Malko et al., 2012; Torrent and Sánchez-Dehesa, 2012; Zhang et al., 2008), Dirac-like cone (with triple degeneracy) (Lu et al., 2016), double Dirac cone (with quadruple degeneracy) (Chen et al., 2014; Li et al., 2014; Li and Mei, 2015; Sakoda, 2012) and semi-Dirac points (Banerjee et al., 2009; Zhai et al., 2011). When the scattering units are arranged in triangular or honeycomb lattices, there usually exists a Dirac cone with twofold degeneracies at the highly symmetric corner in the Brillouin region (Lu et al., 2014; Malko et al., 2012; Torrent and Sánchez-Dehesa, 2012; Zhang et al., 2008) and double Dirac cone (Chen et al., 2014; Li et al., 2014; Li and Mei, 2015; Sakoda, 2012) at the center. Furthermore, Dirac-like cones with a three-fold degeneracy of two linear dispersion bands and an additional flat band were also observed in the hexagonal BZ center (Lu et al., 2017). A semi-Dirac cone refers to a peculiar type of a dispersion relation that is linear along the symmetry line but quadratic in the perpendicular direction, and usually appears in anisotropic phononic crystals (Banerjee et al., 2009; Zhai 
et al., 2011). Studies on Dirac cones have greatly promoted the development of topological acoustic states and topological insulators. However, it is noted that the frequencies of Dirac cones are all fixed when the whole architecture is determined. Although sub-wavelength and multiple Dirac cones were developed recently (Dai et al., 2017; Yves et al., 2017), their tunability remains unresolved. Therefore, it is important to establish a novel system to realize a tunable Dirac cone.

It is known that the band structure can be tuned by changes of geometrical parameters via pattern transformations such as induced instability and geometrical operations (Wang et al., 2014; Yu et al., 2013). In this paper, we proposed twodimensional acoustic metamaterials with matryoshka structure to achieve tunable Dirac cones and topological spin states both via changing the microstructure with rotation of the scatterers. The matryoshka structure is usually composed of concentric circular scattering units, which can obtain multiple band gaps owing to the local resonances (Elford et al., 2011). When we arrange these concentric circular scattering units in honeycomb lattices, Dirac points can be obtained on dispersion curves owing to the high symmetry. By a rotating-scatterer mechanism to break the symmetry, the Diarc cone is split and the topological spin states appear at the band valley. We also demonstrate the existence of a topological transition with opposite Chern numbers as the rotating angle varies, and obtain helical edge states, which are along the interfaces separating the topologically opposite spin states insulators. Moreover, the frequency of the Dirac cone can also be tuned by rotating the inner structure in a double-layer matryoshka structure.

\section{RESULTS AND DISCUSSION}

Figure 1(a) shows the schematic diagram of a unit cell of the single-layer matryoshka structure, where the white part is metal steel, which is considered as a rigid body in the calculation process, and the other parts correspond to air. The whole structure consists of a hexagonal array of slotted cylinders with three splits and can be treated as a Helmholtz resonator with three necks. The structural geometry can be described by the lattice constant $a(=12 \mathrm{~mm})$, the radius of the slotted cylinders $r(=6 \mathrm{~mm})$ and $R(=8 \mathrm{~mm})$, and the split width $W(=0.4 \mathrm{~mm})$. Additionally, the orientation of the slotted cylinder is characterized by the angle $\alpha(0 \leq \alpha \leq \pi / 3)$
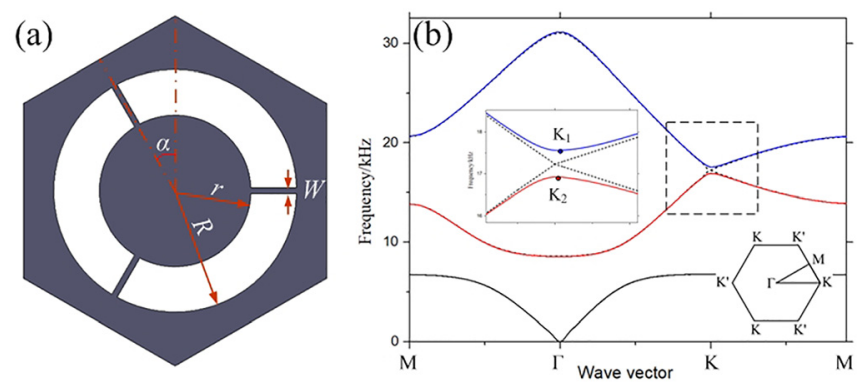

FIG. 1. (Color online) (a) Layout of single-layer matryoshka structure. (b) Calculated band structures, where the solid line refers to the dispersion relation of the structure with $\alpha=\pi / 9$, and the dashed line refers to the dispersion relation of the structure with $\alpha=\pi / 6$. The insert small figure shows the details of band structure near the Dirac cone. with respect to the vertical direction. In this paper, all calculations were carried out using COMSOL Multiphysics finiteelement method (FEM) software. During the calculations, both the air and steel were considered, and their parameters were density $\rho=1.25 \mathrm{~kg} / \mathrm{m}^{3}$, sound velocity $C=343 \mathrm{~m} / \mathrm{s}$ for the air background, and density $\rho=7800 \mathrm{~kg} / \mathrm{m}^{3}$, sound velocity $C=5100 \mathrm{~m} / \mathrm{s}$ for the steel structure. Owing to the large difference between the acoustic impedance, the shear deformation of steel was neglected. In calculations, the triangle element mesh is used. It is worth noting that, in order to ensure the accuracy of the calculation, the maximum element size of the triangle mesh is far less than $1 / 6$ of the shortest wavelength in calculated rage of frequencies.

As shown in Fig. 1(a), it is noted that when $\alpha=\pi / 6$, the whole structure has $C_{3 \mathrm{v}}$ symmetry consistent with a hexagonal lattice, which has both a threefold rotational symmetry and three mirrors. When the internal steel structure rotates by a certain angle, $\alpha$ is not equal to $\pi / 6$, and the symmetry of the whole structure reduces to $C_{3}$ and only has threefold rotational symmetry. The band structures of the single-layer matryoshka structure with $\alpha=\pi / 6$ (dash line) and $\alpha=\pi / 9$ (solid line) are shown in Fig. 1(b). A Dirac cone (at $16.924 \mathrm{kHz})$ appeared at the inequivalent hexagonal corners $K\left(K^{\prime}\right)$ of the first BZ for the single-layer matryoshka structure with $\alpha=\pi / 6$, owing to the mirror symmetry protection. For $\alpha=\pi / 9$, the Dirac cone at point $\mathrm{K}\left(\mathrm{K}^{\prime}\right)$ splits and forms a pair of frequency extrema in the band structure owing to a symmetry mismatch between the steel structure and hexagonal lattice, as shown in Fig. 1(b). These two frequency extrema are respectively marked with valley states $K_{1}$ (at $16.924 \mathrm{kHz}$ ) and $K_{2}$ (at $17.563 \mathrm{kHz}$ ), and form a complete band gap.

Figure 2 shows the curves of two frequency extreme points varying with angle $\alpha$, where the blue line represents valley states $K_{1}$ and red line represents valley states $K_{2}$. The band gap structure around the Dirac cone went from open to closed to open again when the rotating angle $\alpha$ was changed

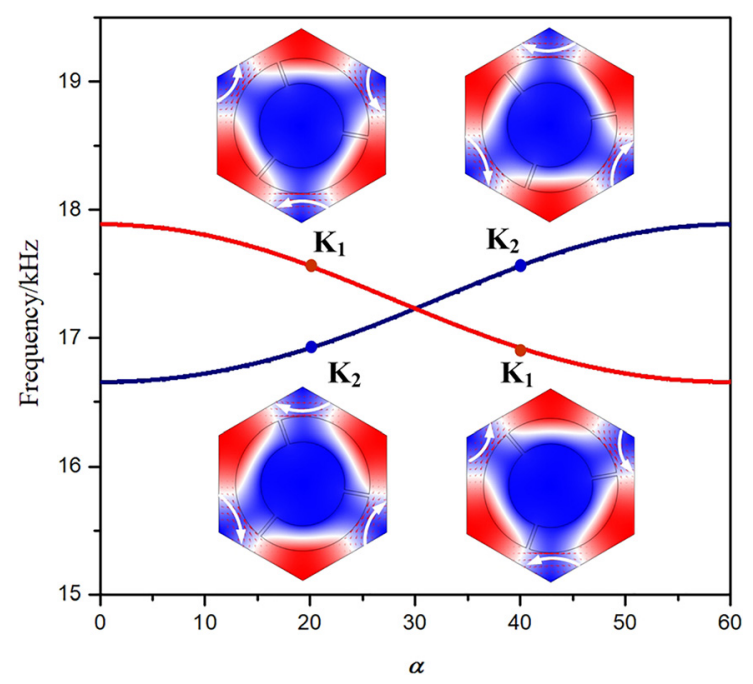

FIG. 2. (Color online) Band-edge frequencies depicted for the acoustic system with different rotation angle $\alpha$, where the color illustrations show the distribution of absolute sound pressure at the valley states $K_{1}$ and $K_{2}$ for $\alpha=\pi / 9$ and $\alpha=2 \pi / 9$. 
from 0 to $\pi / 3$. For $\alpha=\pi / 6$, the band gap was closed, and the band gap was widest for $\alpha=0$ or $\pi / 3$. The color illustrations in Fig. 2 give the distribution of the absolute sound pressure at the valley states $K_{1}$ and $K_{2}$ for $\alpha=\pi / 9$ and $\alpha=2 \pi / 9$. When the rotating angle $\alpha=\pi / 9$, the Dirac cone split and formed two valley states $K_{1}$ and $K_{2}$ owing to the loss of mirror symmetry. The pressure distributions suggested that the two valley states had an opposite vortex in the corner point of hexagonal unit cell: the valley state $K_{1}$ at the upper edge of the band gap had a clockwise chiral vortex, and the valley state $K_{2}$ at the lower edge of the band gap had an anticlockwise chiral vortex. When the rotating angle $\alpha=2 \pi / 9$, the two topological valley states exchanged their positions at the edge of the band gap. For the upper edge of the band gap, the valley state was occupied by $K_{2}$ with an anticlockwise chiral vortex, and the valley state $K_{1}$ with a clockwise chiral vortex appeared at the lower edge. The whole band structure was reversed when the rotation angle $\alpha$ passed through $\pi / 6$, and this inversion process excited the topological spin states and topological phase transitions.

To better understand these topological phase transitions, a nonzero valley Chern number was introduced in this paper. Using the $k \bullet p$ perturbation method, the topological phase transition could be captured by the continuum Hamiltonian (Lu et al., 2016),

$$
H_{K}(\delta \boldsymbol{k})=V_{D} \delta k_{x} \sigma_{x}+V_{D} \delta k_{y} \sigma_{y}+m V_{D}^{2} \sigma_{z},
$$

where $V_{D}$ is the Dirac velocity of the conical dispersion at $\alpha=\pi / 6 . \delta \boldsymbol{k}$ is the deviation of momentum from the valley center $K . \sigma_{i}$ are the Pauli matrices of the vortex pseudospins. The effective mass characterizes two different valley Hall insulators separated by the Dirac semimetal phase with $m=0$ in the phase diagram,

$$
m=\frac{\left(\omega_{K_{1}}-\omega_{K_{2}}\right)}{2 V_{D}^{2}} .
$$

The effective mass is related to the bandwidth, so the effective Hamiltonian is strongly dependent on the rotation angle $\alpha$. Additionally, the nontrivial local Berry curvature centered at the $K$ valley can be calculated by using the eigenvector from the effective Hamiltonian (Zhang et al., 2011),

$$
\Omega_{k}(\delta \boldsymbol{k})=\frac{m V_{D}}{2\left(\delta \boldsymbol{k}^{2}+m^{2} V_{D}^{2}\right)^{3 / 2}} .
$$

The topological charges of the first band can be calculated by integrating the local Berry curvature (Martin et al., 2008; Semenoff et al., 2008),

$$
C_{k}=\frac{1}{2 \pi} \int \Omega_{k}(\delta \boldsymbol{k}) d s=\frac{1}{2} \operatorname{sgn}(m) .
$$

The Chern number of $K^{\prime}$ can be derived from timereversal symmetry and has opposite signs,

$$
C_{k^{\prime}}=-C_{k}=\frac{1}{2} \operatorname{sgn}(m)
$$

The valley Chern number can be obtained as

$$
C_{v}=C_{k}-C_{k^{\prime}}
$$

With the rotating angle $0 \leq \alpha<\pi / 6$, the valley Chern number is 1 , and the Chern number is -1 for $\pi / 6<\alpha \leq \pi / 3$. Thus, the topological phase transition occurs with the change of the valley Chern number at the rotating angle $\alpha=\pi / 6$, which predicts edge states propagating along the interface between two hexagonal lattices with a symmetric rotating angle.

To verify the edge states, a super cell structure was built, as shown in Fig. 3. The super cell consisted of three parts: I-II-I. Part I had units with the rotating angle $\alpha=\pi / 9$, and part II had units with the rotating angle $\alpha=2 \pi / 9$. There were two kinds of interfaces, I-II and II-I, in this super cell structure. In the calculation process, a Floquent periodic boundary condition was applied at the right and left boundaries of the super cell structure. Meanwhile, perfectly matched layers (PML) were chosen at the upper and lower edges of the structure to represent an infinite domain of air. The band structure of the whole super cell and pressure
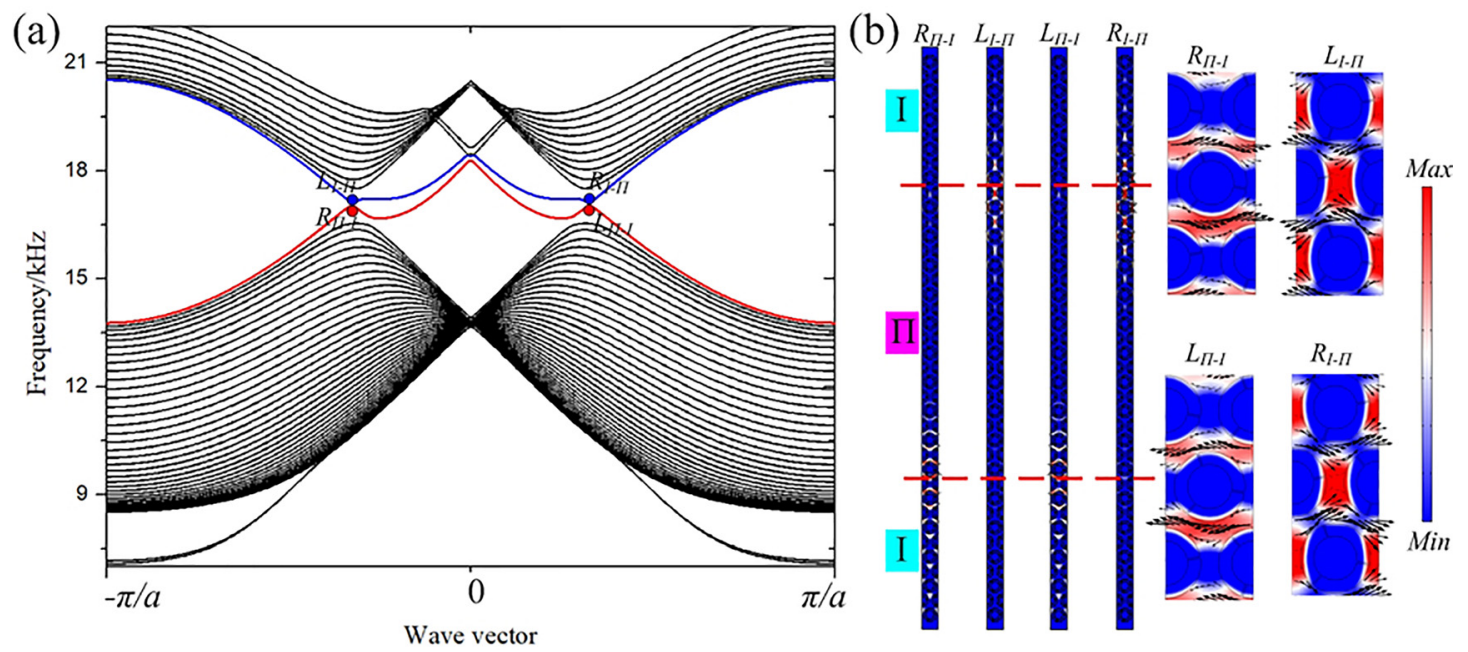

FIG. 3. (Color online) (a) Interface dispersions (dark lines) evaluated for I-II-I type superlattice structure, where the grey line corresponds to the bulk states and the colored lines correspond to the edge states. (b) Pressure distributions of the four eigenstates marked with $L_{I-I I}, R_{I-I I}, L_{I I-I}, R_{I I-I}$. 


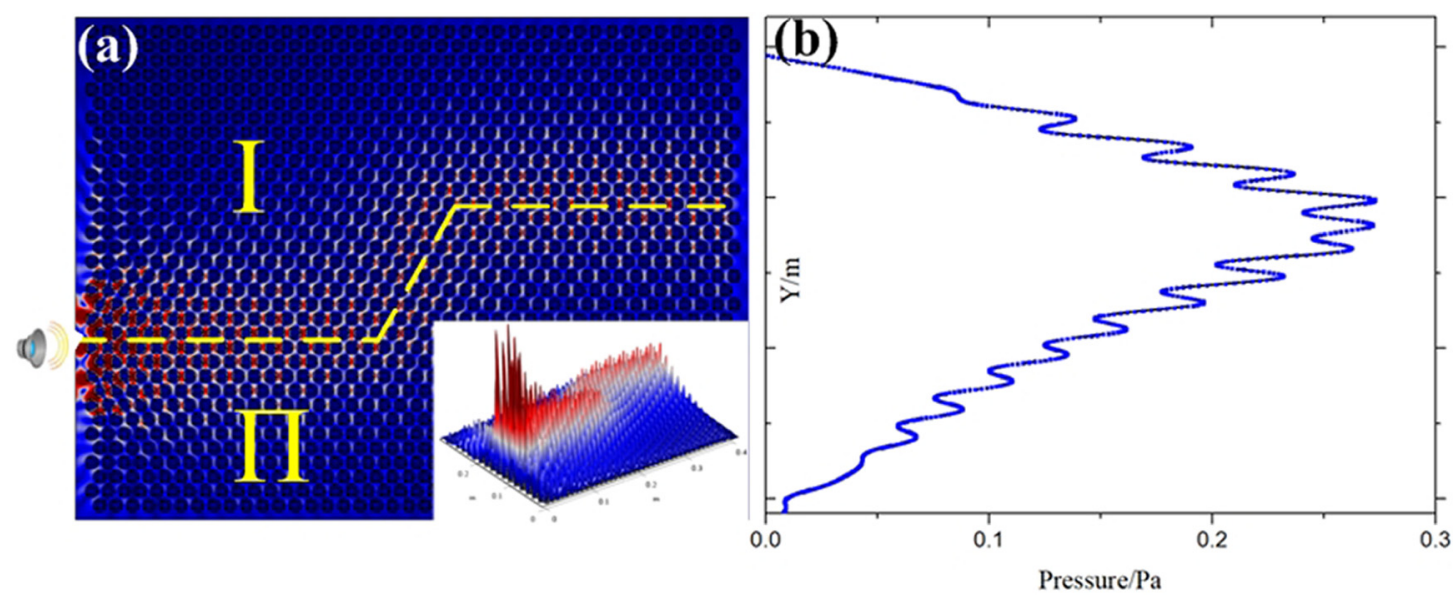

FIG. 4. (Color online) (a) Pressure distributions of a zigzag bending channel at $17300 \mathrm{~Hz}$, and the insert small figure shows the height map of sound pressure. (b) Pressure distribution at the right boundary along the $y$ axis.

distributions at special frequencies are shown in Fig. 3. The red and blue lines in Fig. 3(a) indicate the interface dispersions and the black lines correspond to the bulk states. The pressure distributions of the four eigenstates as the edge states [marked in Fig. 3(a)] indicated the one-way transmission at the interface between the different unit cells, as shown in Fig. 3(b). The edge states existed in the super cell system and were respectively marked as $L_{I-I I}, R_{I-I I}, L_{I I-I}, R_{I I-I}$, where $L$ and $R$ represent two opposite propagation directions, and $I-I I$ and $I I-I$ represent two kinds of interfaces. For instance, the sound wave of the $R_{I-I I}$ state only transmits to the right at the I-II interface. It is noted that the edge states are helical edge modes owing to the valley pseudospin by analogy with the electronic system (Bernevig et al., 2006). The results suggested that the sound can efficiently transmit along the topological interfaces owing to the helical edge states, which is caused by the opposite Chern numbers and topological spin states at the two sides of the interface. This robust transmission was not disturbed by external defects and disturbances.

Furthermore, a zigzag bending channel, which was separated by part I with the rotating angle $\alpha=\pi / 9$, and part II with the rotating angle $\alpha=2 \pi / 9$, was built to examine the transmission at the edge states as shown in Fig. 4. A point source at frequency $17300 \mathrm{~Hz}$ was applied at the left boundary around the interface between two parts, and the pressure distributions are shown in Fig. 4(a). Figure 4(b) shows the pressure distribution at the right boundary along the $y$ axis and suggests that the transmission wave at the exit was concentrated at the topological interface between two parts. The sound travelled smoothly along the zigzag path despite encountering two sharp corners. The altitude maps [see inset of Fig. 4(a)] show that the transmission was highly efficient and exhibited good immunity to strong backscattering caused by bending in the transmission path. From this, we observed that we could construct an arbitrary topological interface by rotating the internal scattering units, and then realized the efficient transmission of sound waves along arbitrary paths.

Based on a single-layer matryoshka structure, the topological spin states and topological insulators were designed by rotating the scattering units. Subsequently, a double-layer matryoshka structure was designed to obtain a tunable Dirac cone. As shown in Fig. 5, the double-layer matryoshka structure had two layers of slotted cylinders. The whole geometry could be described by the lattice constant $a(=12 \mathrm{~mm})$, the radius of the outer cylinders $r_{1}(=3 \mathrm{~mm})$ and $R_{1}(=4 \mathrm{~mm})$, and the radius of the inner cylinders $r_{2}(=1.5 \mathrm{~mm})$ and $R_{2}$ $(=2.5 \mathrm{~mm})$, and the split width $W(=0.8 \mathrm{~mm})$. Orientation of the outer and inner cylinder was characterized respectively by the angle $\alpha(0 \leq \alpha \leq \pi / 3)$ and $\beta(0 \leq \alpha \leq \pi / 3)$ with respect to the vertical direction. The band structures of the double-layer matryoshka structure with $\alpha=\pi / 6, \beta=\pi / 9$ (dashed line) and $\alpha=\pi / 9, \beta=\pi / 9$ (solid line) are shown in Fig. 5(b). Similar to single-layer structure with $\alpha=\pi / 6$, the double-layer matryoshka structure also exhibited a Dirac cone at $15.306 \mathrm{kHz}$, and when the outer layer was rotated, the Dirac cone at point $K\left(K^{\prime}\right)$ split and formed a pair of topological spin states. It is also noted that the Dirac cone still existed although the symmetry of the inner structure was mismatched with the hexagonal lattice.

Figure 6(a) shows the curves of the frequencies of two topological spin states varying with angle $\alpha$. Compared with a single layer, the band gap structures also changed from open to closed to open again when angle $\alpha$ was varied from 0 to $\pi / 3$, and the only difference was that the curves of the double layer was asymmetrical owing to the mirror
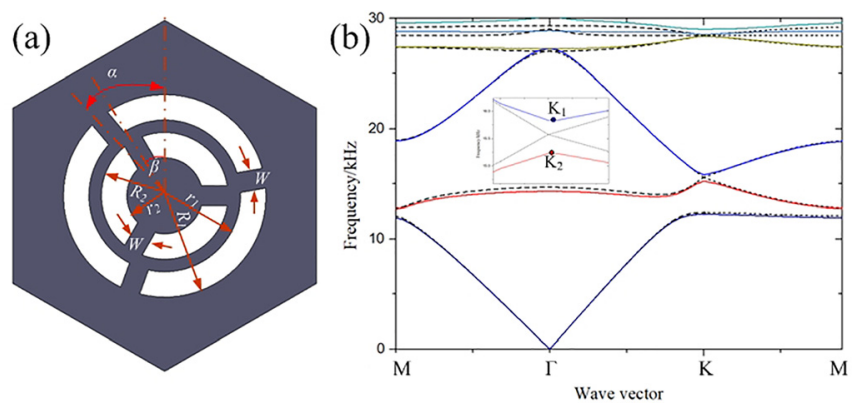

FIG. 5. (Color online) (a) Layout of double-layer matryoshka structure. (b) Calculated band structures. The solid line refers to dispersion relation of the structure with $\alpha=\pi / 9, \beta=\pi / 9$, and the dashed line refers to dispersion relation of the structure with $\alpha=\pi / 6, \beta=\pi / 9$. The insert small figure shows the details of band structure near the Dirac cone. 

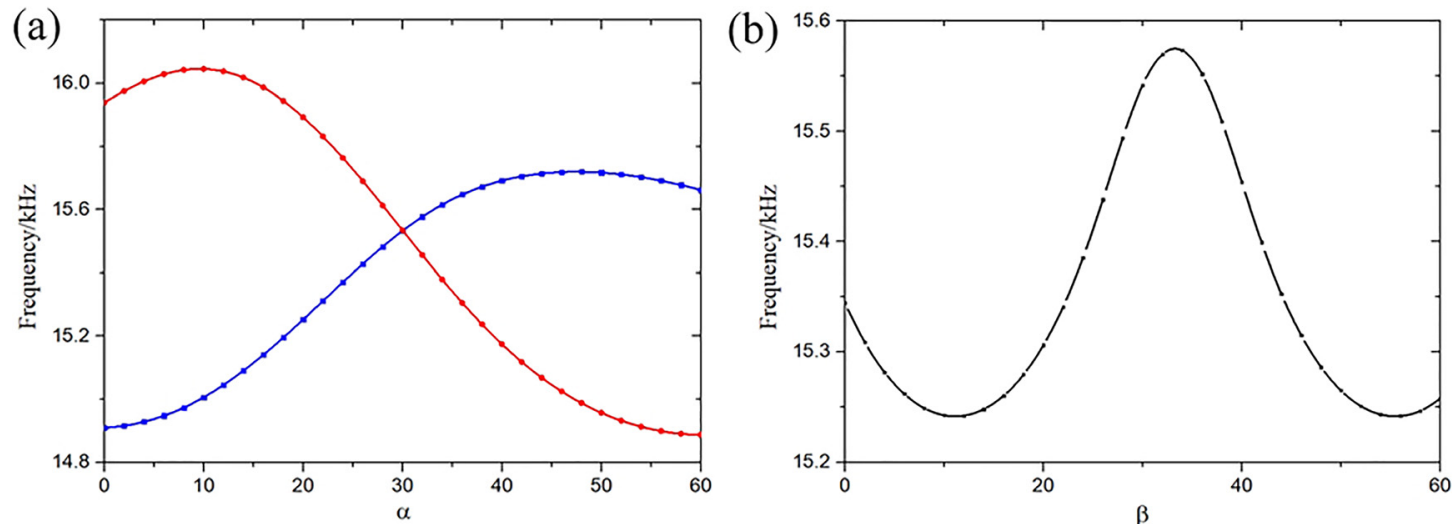

FIG. 6. (Color online) (a) Frequencies of two topological spin states varying with angle $\alpha$. (b) Frequencies at Dirac cone with different $\beta$ of the double-layer matryoshka structure.

symmetry mismatch between the inner and outer structures. The frequencies at the Dirac cone with different $\beta$ are given in Fig. 6(a). A tunable Dirac cone was obtained when rotating the inner layer. All results suggested that the Dirac cone only depended on the mirror symmetry of the outer structure. Hence, no matter how the inner structure was rotated, the band gap will close and form the Dirac cone when the symmetry of the outer structure matches with the hexagonal lattice, and the band gap opens when the outer symmetry is mismatched. Moreover, the frequency position of the Dirac cone can be affected by $\beta$, and it can be tuned by rotating the inner structure. When rotating the inner structure, the position of Dirac cone will be tuned due to the change of coupling between the inner and outer structure. It can be found that the rotation operation is not only used to reduce symmetry to achieve degeneracy of Dirac cone, but also can realize tunable Dirac cones via changing the microstructure for double-layer matryoshka structure. In addition, the tunable frequency rage can also be widened by optimizing the structure parameters such as split width and interlayer spacing. If the inner and outer structure are both made into moving parts, the whole system is very practical in use.

\section{CONCLUSIONS}

In this paper, matryoshka structures are proposed to achieve a Dirac cone and topological phase transitions. The results suggest that a Dirac cone appears at the inequivalent hexagonal corners $K\left(K^{\prime}\right)$ for the single-layer matryoshka structure with $\alpha=\pi / 6$, owing to the mirror symmetry protection. Additionally, the topological spin states and topological phase transitions with opposite Chern numbers are achieved by a rotating-scatterer mechanism to break the mirror symmetry. Movement of the acoustic edge states are further observed along the interfaces separating the topologically different valley insulators, and their transmission properties are determined in a zigzag bending channel. Moreover, a double-layer matryoshka structure is designed to obtain a tunable Dirac cone by rotating the inner layer. When rotating the inner structure, the position of Dirac cone will be tuned due to the change of coupling between the inner and outer structure. The results suggest these novel topological structures with a tunable Dirac cone offer the potential design of novel tunable acoustic topological materials and devices for practical applications in sound control fields.

\section{ACKNOWLEDGMENT}

We acknowledge project supported by the National Natural Science Foundation of China (Grant Nos. 11602269 and 11802213), the Strategic Priority Research Program of the Chinese Academy of Sciences (Grant No. XDB22040301) and the Research Program of Beijing (Grant Nos. Z161100002616034 and Z171100000817010).

Banerjee, S., Singh, R. R. P., Pardo, V., and Pickett, W. E. (2009). "Tightbinding modeling and low-energy behavior of the semi-Dirac point," Phys. Rev. Lett. 103(1), 016402.

Bernevig, B. A., Hughes, T. L., and Zhang, S. C. (2006). "Quantum spin hall effect and topological phase transition in HgTe quantum wells," Science 314, 1757-1761.

Chen, Z.-G., Ni, X., Wu, Y., He, C., Sun, X.-C., Zheng, L.-Y., Lu, M.-H., and Chen, Y.-F. (2014). "Accidental degeneracy of double Dirac cones in a phononic crystal," Sci. Rep. 4(1), 4613.

Dai, H., Xia, B., and Yu, D. (2017). "Dirac cones in two-dimensional acoustic metamaterials," J. Appl. Phys. 122(6), 065103.

Elford, D. P., Chalmers, L., Kusmartsev, F. V., and Swallowe, G. M. (2011). "Matryoshka locally resonant sonic crystal," J. Acoust. Soc. Am. 130(5), 2746-2755.

Fleury, R., Khanikaev, A. B., and Alù, A. (2016). "Floquet topological insulators for sound," Nat. Commun. 7, 11744.

Fleury, R., Sounas, D. L., Sieck, C. F., Haberman, M. R., and Alu, A. (2014). "Sound isolation and giant linear nonreciprocity in a compact acoustic circulator," Science 343(6170), 516-519.

Häusler, W., and Egger, R. (2009). "Artificial atoms in interacting graphene quantum dots," Phys. Rev. B 80(16), 161402.

He, C., Li, Z., Ni, X., Sun, X.-C., Yu, S.-Y., Lu, M.-H., Liu, X.-P., and Chen, Y.-F. (2016). "Topological phononic states of underwater sound based on coupled ring resonators," Appl. Phys. Lett. 108(3), 031904.

He, C., Ni, X., Ge, H., Sun, X.-C., Chen, Y.-B., Lu, M.-H., Liu, X.-P., and Chen, Y.-F. (2016). "Acoustic topological insulator and robust one-way sound transport," Nat. Phys. 12(12), 1124-1129.

Herring, C. (1937). "Accidental degeneracy in the energy bands of crystals," Phys. Rev. 52(4), 365-373.

Khanikaev, A. B., Fleury, R., Mousavi, S. H., and Alù, A. (2015). "Topologically robust sound propagation in an angular-momentum-biased graphene-like resonator lattice," Nat. Commun. 6(1), 8260.

Li, Y., and Mei, J. (2015). "Double Dirac cones in two-dimensional dielectric photonic crystals," Opt. Express 23(9), 12089.

Li, Y., Wu, Y., and Mei, J. (2014). "Double Dirac cones in phononic crystals," Appl. Phys. Lett. 105(1), 014107.

Lu, J., Qiu, C., Xu, S., Ye, Y., Ke, M., and Liu, Z. (2014). "Dirac cones in two-dimensional artificial crystals for classical waves," Phys. Rev. B 89(13), 134302. 
Lu, J., Qiu, C., Ye, L., Fan, X., Ke, M., Zhang, F., and Liu, Z. (2016). "Observation of topological valley transport of sound in sonic crystals," Nat. Phys. 13(4), 369-374.

Ma, G., and Sheng, P. (2016). "Acoustic metamaterials: From local resonances to broad horizons," Sci. Adv. 2(2), e1501595.

Malko, D., Neiss, C., Viñes, F., and Görling, A. (2012). "Competition for graphene: Graphynes with direction-dependent Dirac cones," Phys. Rev. Lett. 108(8), 086804.

Martin, I., Blanter, Y. M., and Morpurgo, A. F. (2008). "Topological confinement in bilayer graphene," Phys. Rev. Lett. 100(3), 036804.

Novoselov, K. S., Geim, A. K., Morozov, S. V., Jiang, D., Katsnelson, M. I., Grigorieva, I. V., Dubonos, S. V., and Firsov, A. A. (2005). "Two-dimensional gas of massless Dirac fermions in graphene,” Nature 438(7065), 197-200.

Peng, Y.-G., Qin, C.-Z., Zhao, D.-G., Shen, Y.-X., Xu, X.-Y., Bao, M., Jia, H., and Zhu, X.-F. (2016). "Experimental demonstration of anomalous Floquet topological insulator for sound," Nat. Commun. 7, 13368.

Sakoda, K. (2012). "Double Dirac cones in triangular-lattice metamaterials," Opt. Express 20(9), 9925.

Semenoff, G. W., Semenoff, V., and Zhou, F. (2008). "Domain walls in gapped graphene," Phys. Rev. Lett. 101(8), 087204.

Torrent, D., and Sánchez-Dehesa, J. (2012). "Acoustic analogue of graphene: Observation of Dirac cones in acoustic surface waves," Phys. Rev. Lett. 108(17), 174301.

Wang, P., Casadei, F., Shan, S., Weaver, J. C., and Bertoldi, K. (2014). "Harnessing buckling to design tunable locally resonant acoustic metamaterials," Phys. Rev. Lett. 113, 014301.

Wang, P., Lu, L., and Bertoldi, K. (2015). "Topological phononic crystals with one-way elastic edge waves," Phys. Rev. Lett. 115, 104302.

Wei, Q., Tian, Y., Zuo, S.-Y., Cheng, Y., and Liu, X.-J. (2017). "Experimental demonstration of topologically protected efficient sound propagation in an acoustic waveguide network," Phys. Rev. B 95(9), 094305.

Wen, X., Qiu, C., Lu, J., He, H., Ke, M., and Liu, Z. (2018). "Acoustic Dirac degeneracy and topological phase transitions realized by rotating scatterers,” J. Appl. Phys. 123(9), 091703.
Wu, Y., Yang, M., and Sheng, P. (2018). "Perspective: Acoustic metamaterials in transition," J. Appl. Phys. 123(9), 090901.

Xia, B., Liu, T., Huang, G., Dai, H., Jiao, J., Zang, X., Yu, D., Zheng, S., and Liu, J. (2017). "Topological phononic insulator with robust pseudospin-dependent transport," Phys. Rev. B 96, 094106.

Xia, B., Wang, G., and Zheng, S. (2019). "Robust edge states of planar phononic crystals beyond high-symmetry points of Brillouin zones," J. Mech. Phys. Solids 124, 471.

Xia, B., Zheng, S., Liu, T., Jiao, J., Chen, N., Dai, H. Q., and Liu, J. (2018). "Observation of valley-like edge states of sound at a momentum away from the high-symmetry points," Phys. Rev. B 97, 155124.

Yang, Z., Gao, F., Shi, X., Lin, X., Gao, Z., Chong, Y., and Zhang, B. (2015). “Topological acoustics," Phys. Rev. Lett. 114(11), 114301.

Yu, K., Chen, T., Wang, X., and Zhou, A. (2013). "Large band gaps in phononic crystal slabs with rectangular cylinder inclusions parallel to the slab surfaces," J. Phys. Chem. Solids 74, 1146.

Yves, S., Fleury, R., Lemoult, F., Fink, M., and Lerosey, G. (2017). "Topological acoustic polaritons: Robust sound manipulation at the subwavelength scale," New J. Phys. 19(7), 075003.

Zhai, F., Mu, P., and Chang, K. (2011). "Energy spectrum of Dirac electrons on the surface of a topological insulator modulated by a spiral magnetization superlattice," Phys. Rev. B 83(19), 195402.

Zhang, F., Jung, J., Fiete, G. A., Niu, Q., and MacDonald, A. H. (2011). "Spontaneous quantum hall states in Chirally stacked few-layer graphene systems," Phys. Rev. Lett. 106(15), 156801.

Zhang, X., and Liu, Z. (2008). "Extremal transmission and beating effect of acoustic waves in two-dimensional sonic crystals," Phys. Rev. Lett. 101(26), 264303.

Zhang, Y., Tan, Y.-W., Stormer, H. L., and Kim, P. (2005). "Experimental observation of the quantum Hall effect and Berry's phase in graphene," Nature 438(7065), 201-204.

Zhang, Z., Wei, Q., Cheng, Y., Zhang, T., Wu, D., and Liu, X. (2017). "Topological creation of acoustic pseudospin multipoles in a flow-free symmetry-broken metamaterial lattice," Phys. Rev. Lett. 118(8), 084303. 